\title{
Mandibular lateral translation during symmetric mandibular function in relation to patterns of intracapsular derangement of TMJ
}

\author{
K. Suzuki, T. Mito, K. Ishizaki, S. Sato \\ Division of Orthodontics, Department of Craniofacial Growth and Development Dentistry, \\ Kanagawa Dental College, Yokosuka, Japan
}

Received December 2, 2008; Accepted December 22, 2008

Purpose: The purposes of this study were to examine the occurrence of different types of mandibular lateral translation $(\triangle \mathrm{YMLT})$ using condylography and magnetic resonance imaging (MRI), to investigate the relationship between $\triangle \mathrm{YMLT}$ and the disunion pattern in intracapsular derangement patients with $\triangle \mathrm{YMLT}$, and to consider the three-dimensional therapeutic position of the condyle in the mandibular fossa.

Material and methods: Sixty-two patients with $\triangle \mathrm{YMLT}$, or side shift, during symmetrical mandibular movements were analyzed with computerized condylography and MRI to determine the occurrence of different types of MLT and the relationship between the $\triangle \mathrm{YMLT}$ and the intracapsular derangement pattern.

Results: Of a total of 62 subjects, we classified 43 (69.4\%), $12(9.4 \%)$, and $7(11.2 \%)$ of them as $\triangle$ YMLT type, closed lock type, and excessive condylar rotation type, respectively. There was a high prevalence of $\Delta$ YMLT for subjects who had one side antero-medial (including medial sideway) and the other side antero-lateral disk displacement. The directions of $\Delta$ YMLT and disk displacement coincided in $95.3 \%$ of cases; this means recapturing of the displaced disk by the condyle in lower joint translation.

Conclusion: Results indicate that mandibular lateral displacement causes the condyle to be displaced in the same direction, and the disk displacement to the opposite side; hence it creates $\triangle \mathrm{YMLT}$ in the disk displacement direction. On the basis of these findings, it is concluded that the $\triangle$ YMLT is a useful indicator for early detection of intracapsular derangement and that the $\triangle$ YMLT allows estimation of the therapeutic condylar position.

Keywords: Delta Y shift, over rotation, closed lock, intracapsular derangement

Correspondence: Prof. Dr. Sadao Sato, Division of Orthodontics, Department of Craniofacial Growth and Development Dentistry, Kanagawa Dental College, 82 Inaoka-cho, Yokosuka 238-8580, Japan. E-mail: satos@kdcnet.ac.jp

\section{Introduction}

Many temporomandibular disorder (TMD) patients show intracapsular derangement of the temporomandibular joint (TMJ) and are diagnosed as having disunion of the condyledisk assembly $[4,5]$. A high incidence of disunion in patients with TMD was reported in comprehensive studies using arthrography and magnetic resonance imaging (MRI) $[15,17,9]$. Studies of disunion are considered to be essential for the understanding of TMD [3]. Patients with TMD are also reported to show a characteristic condylar path in the sagittal plane in condylographic tracing $[18,19]$. The analysis of condylar path during mandibular movements is highly significant for the examination and diagnosis of internal derangement of TMJ [19].

In patients with disunion, disturbance in sagittal tracing of the condyle during opening and closing movements of the mandible is a common event depending on the patterns of disunion and reducibility $[18,19]$. Interestingly, in transversal tracing of condylography, we often encounter complex lateral translation of the condyle during opening and closing movements. It has been reported that disunion occurs not only anteriorly, but also rotational and sideways $[7,8]$. The pattern of disunion is important for the diagnosis and treatment planning of internal derangement of TMJ. It appears that disturbance in the lateral translation of the condyle is closely related to the patterns of disunion [6]. Thus, the analysis of lateral translation may provide important clues to help identify disunion patterns in patients with intracapsular derangement.

The phenomenon of mandibular lateral translation (MLT) has been described in the pantographic studies of mandibular movement, which included immediate side shift, early side shift, distributed side shift, and progressive side shift $[1,2]$. These lateral translations occur in asymmetric mandibular movement like laterotrusion. However, lateral translation during symmetrical mandibular movements like open-close or protrusion-retrusion movement has not been described in the literature. Only Fushima et al. [6] described this phenomenon in relation to articular disk displacement with reduction. 
Previously, we proposed that the lateral translation during symmetrical mandibular movement is referred as $\triangle$ YMLT [14], because of condylar shift along the Y axis in a coordinated three-dimensional recording of mandibular movement. Therefore, $\triangle$ YMLT can be defined as mandibular lateral translation during symmetrical mandibular movement or symmetrical function.

The purposes of this study were 1) to examine the occurrence of different types of MLT using condylography and MRI, 2) to investigate the relationship between the $\Delta$ YMLT and the disunion pattern in the intracapsular derangement patients with $\triangle \mathrm{YMLT}$, and 3) to consider the three-dimensional therapeutic position of the condyle in the mandibular fossa. We hypothesized that $\triangle \mathrm{YMLT}$ is one of the important indicators for internal derangement of TMJ and position and direction of $\triangle$ YMLT may indicate types of articular disk displacement and therapeutic condylar position.

\section{Materials and methods}

\section{Subjects}

Sixty-two patients ( 17 males and 45 females), diagnosed with disk displacement based on clinical examination, sagittal condylar path analysis and MRI assessment, were selected randomly for participation in this study. The mean age of the patients was 23 years 4 months (ranged from 12 years 10 months to 55 years 7 months). Three-dimensional condylar paths of the patients were recorded during opening and closing movements of the mandible and analyzed with a computerized condylography (Cadiax III ${ }^{\circledR}$, GAMMA GmbH, Austria) system.

\section{Computerized condylography}

The condylographic system consists of a cranial attachment and a mandibular face bow. A functional clutch was fixed to the labial surface of the lower dentition, and mandibular movements were confirmed to be free of any interference. The mandibular face bow was then connected to the clutch. Two-dimensional condylar movement in the sagittal plane was recorded by digitizers, which were attached to the cranial face-bow and placed over the TMJ region bilaterally [16]. Lateral deviation of the condyle during mandibular functional movement was detected by the stylus attached to the mandibular face-bow.

After positioning the stylus on the hinge axis, condylar movements in both TMJ were recorded three-dimensionally from a reference position (RP) established by using unforced chin point guidance [18]. To investigate the $\triangle$ YMLT of the condyle during opening, the condylar paths in the transversal plane were observed on the condylographic tracing. As shown in Fig. 3, the typical $\triangle$ YMLT indicated medial (inward) and lateral (outward) deviation on the way of opening and closing condylar movement.

\section{Magnetic Resonance Imaging (MRI)}

Bilateral MRI were taken to diagnose the disk displacement patterns. A total of 124 were assessed by MRI. MRI was performed utilizing the 0.5 -T MRI system (RESONA; GE) with an 8-cm-diameter surface coil. T1-weighted multi-sliced images of spin echo (TR: $1000 \mathrm{~ms}$, TE: $25 \mathrm{~ms}$, NEX: 2, FOV: I $5 \mathrm{~cm}$, Matrix: $160 \times 224)$ were obtained in the sagittal plane both in the intercuspal position (ICP) and the maximum opening position of the mandible. In addition to the sagittal aspect, TMJ in ICP was also imaged in the coronal plane. Multi-slices were taken in 4-mm sections at 1-mm intervals. The slices were oriented perpendicular to the long axis of the condyle for sagittal images and parallel to the axis for coronal images. The sagittal images were used to determine the antero-posterior position of the disk in the lateral, central and medial slices. The coronal images were used to determine latero-medial disk displacement. Disk displacement patterns were assessed according to the criteria described previously. In normal joints, the position of the posterior band of a disk is superior to the top of the condylar head in every sagittal slice image. In the coronal plane, the disk covers the superior surface of the condyle, including the medial and lateral poles. Disk displacement patterns were diagnosed based on the previously described method with slight modification as the following criteria:

1. Complete anterior displacement: The disk is displaced anteriorly in lateral, central and medial slices of sagittal images, and the quantity of disk in each slice is nearly identical (Fig. 1). In the coronal plane, the disk is observed in a slice anterior to the condyle.

2. Antero-lateral displacement: The disk is displaced anteriorly in every sagittal slice. The disk quantity is large in lateral slices, but there is little or no disk in medial slices. In coronal images, lateral displacement of the disk is confirmed in a slice anterior to the condyle.

3. Antero-medial displacement: The disk is displaced anteriorly in every sagittal slice. The disk quantity is large in medial slices, but there is little or no disk in lateral slices. In coronal images, medial displacement of the disk is confirmed in a slice anterior to the condyle.

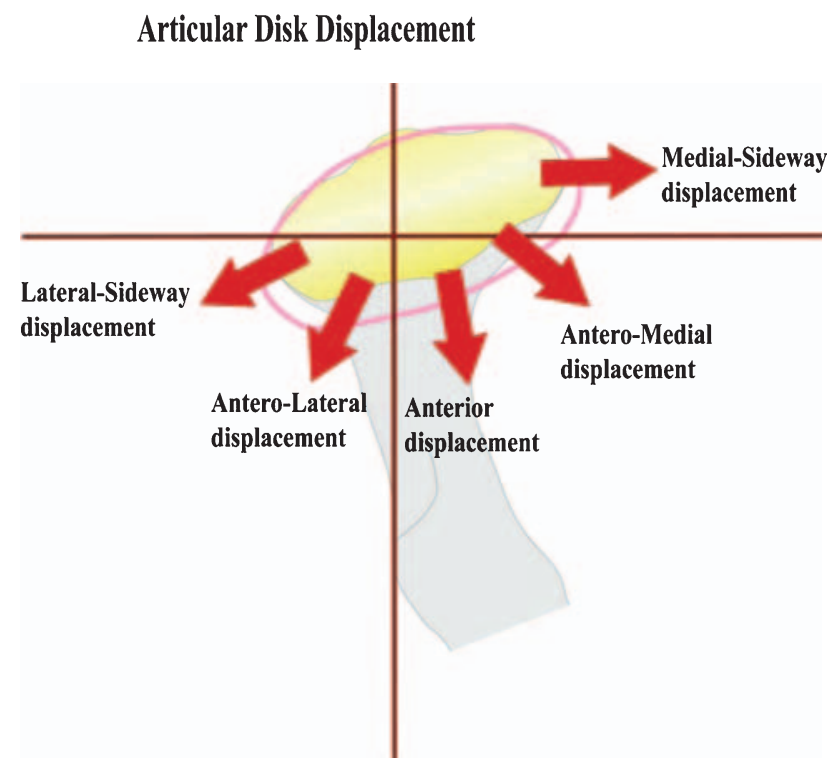

Fig. 1: Patterns of articular disk displacement 
4. Medial sideways displacement: There is no anterior disk displacement in any sagittal image, and medial disk displacement is confirmed in a slice through the condyle in the coronal image. Another feature for assessment is the loss of joint space or an empty fossa in lateral slices of the sagittal image. The disk does not cover the lateral pole of the condyle, and medial folding of the disk inferior to the medial pole of the condyle is frequently observed.

5. Lateral sideways displacement: There is no anterior disk displacement in any sagittal image, and lateral disk displacement is confirmed in a slice through the condyle in the coronal image. The disk does not cover the medial pole of the condyle, and lateral folding of the disk inferior to the lateral pole of the condyle is observed.

In all patients selected in this study, disk reduction or non-reduction was confirmed using sagittal images during maximum opening.

One representative case is shown in Fig. 2 to explain the MRI examination. In this case, the right TMJ exhibited lateral-sideway displacement with reduction based on the findings of no-anterior displacement disk in sagittal images with large disk quantity in lateral slices (Fig. 2a) and the left TMJ showed antero-lateral displacement with reduction based on the finding of quantity of anteriorly displaced disk in sagittal images (Fig. 2b). Coronal images of MRI confirmed these findings (Fig. 2c). Condylographic tracing of the open/ close movement represented the $\triangle$ YMLT in right and left joints (Fig. 3).

\section{Results}

\section{Characterizations of $\Delta Y$ shift}

Observations of lateral translation of the condyle showed that there were three predominant types of condylar deviation. The first type, bilateral $\Delta \mathrm{Y}$ shift occurred within 1 to $7 \mathrm{~mm}$ of translation from the RP during opening movement $(\Delta \mathrm{Y})$. This group showed a reducible articular disk on both sides or at least one side in the MRI study. The second type showed lateral translation with strong limitation of condylar movement and non-reducible articular disk in both sides of the joints (Closed locked type). In the third type, lateral translation

\section{a}
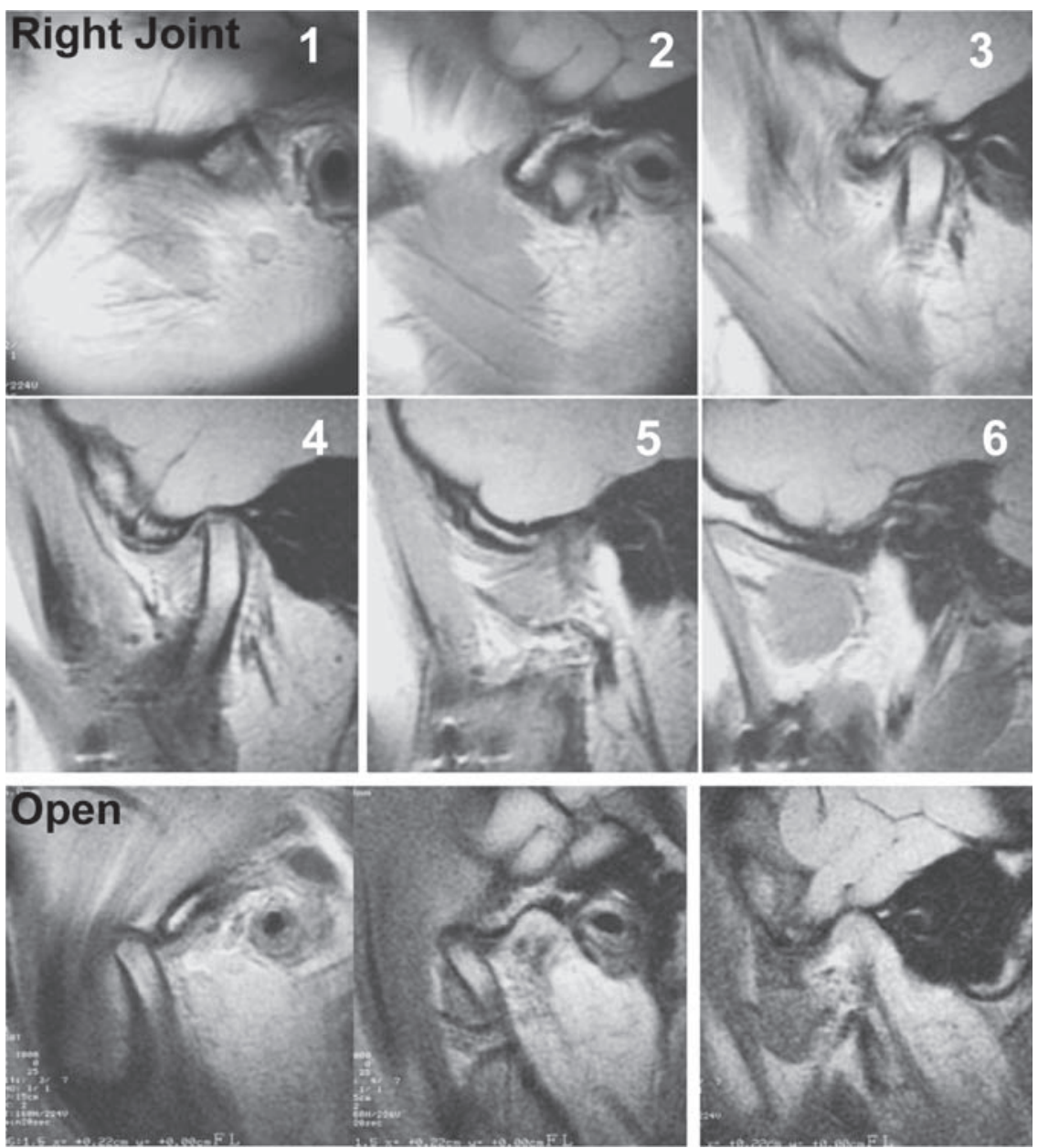

Fig. 2: Evaluation of disk displacement pattern using MRI (a) Sagittal lateral slice (1-3) of right TMJ showed lateral part of the disk, while central (4) and medial $(5,6)$ slices did not show disk displacement. (b) Left TMJ shows anterior disk displacement in lateral $(1-4)$, while medial slice $(5,6)$ did not show anterior disk displacement. Both TMJ were with disk reduction in opening position. (c) Coronal images of MRI showed laterally displaced disk. Together with sagittal and coronal images, it was diagnosed as lateral sideway displacement in right TMJ and antero-lateral disk displacement in left TMJ 
b
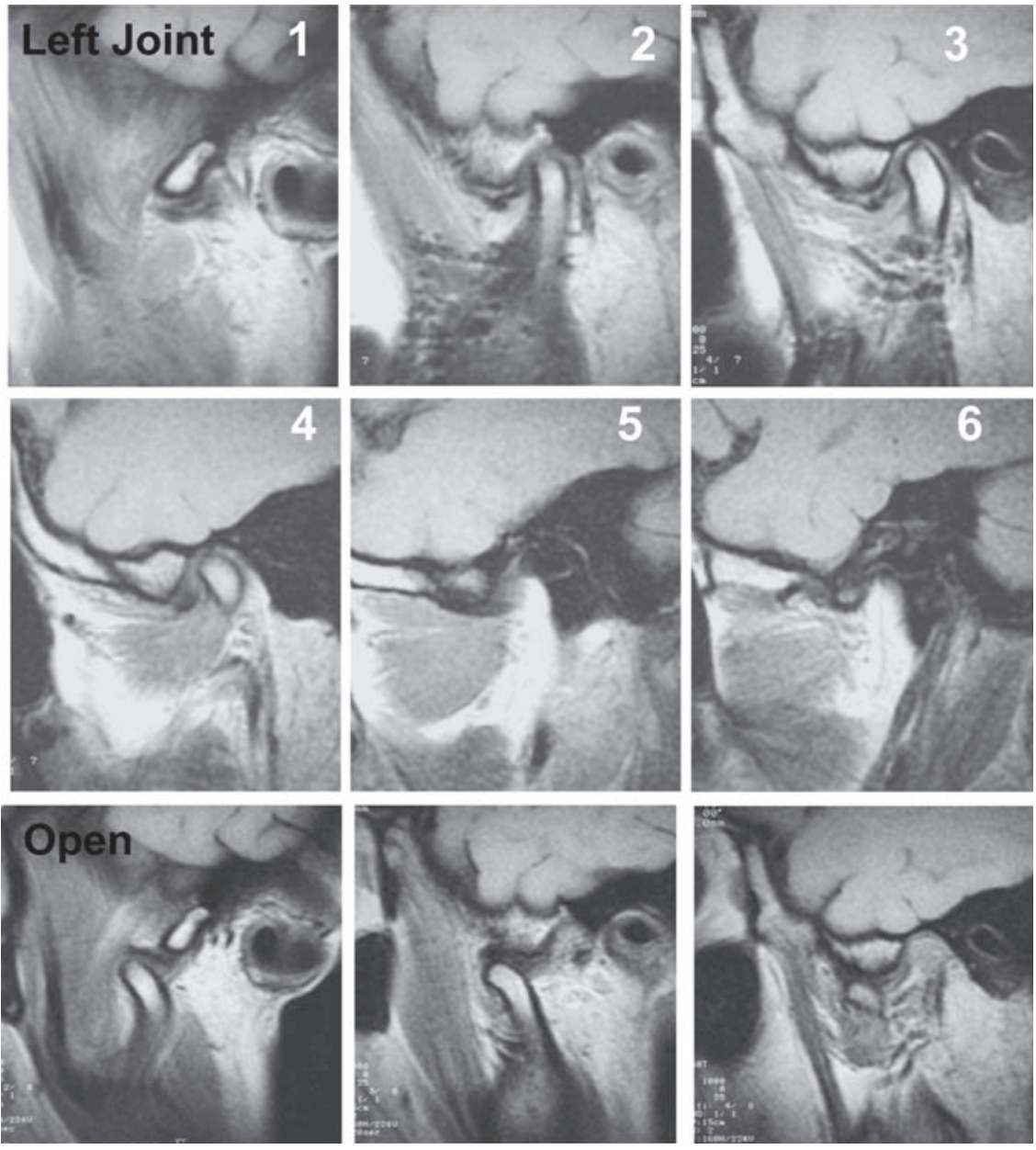

C
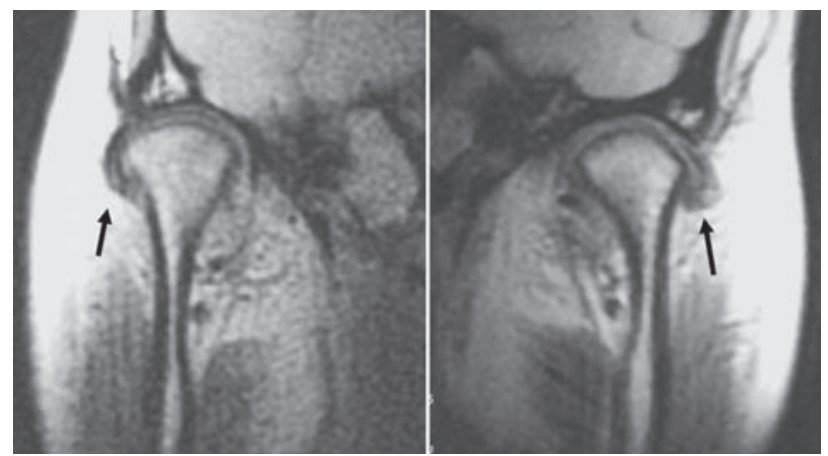

Fig. 2: (Continued)

occurred at a nearly maximum opening position (Excess condylar rotation type). Forty-three of 62 subjects (69.4\%) were classified as $\triangle \mathrm{YMLT}$ shift type, while 12 subjects (19.4\%) were closed lock type, and 7 subjects $(11.2 \%)$ were excessive condylar rotation type (Tab. 1). Therefore, $\Delta \mathrm{Y}$ MLT types of lateral translation was separately analyzed in this study (Fig. 4).

\section{Combination of disk displacement}

There were different combinations of articular disk displacement in the right and left joints (Tab. 2). Majority of joints which had $\triangle$ YMLT behavior showed bilateral reducible disk displacement ( $54.1 \%$ of joints), followed by unilateral reducible disk displacement (13.1\%) and bilateral non-reducible disk displacement (closed lock, 19.4\%).

\section{$\triangle Y M L T$ and disk displacements}

To serve the purpose in this study, we selected the $\Delta$ YMLT group for further analysis. In this group, 70 of 86 joints (81.4\%) showed a reducible articular disk (Tab. 3). Results of MRI analysis showed that the articular disk displacement in the $\triangle \mathrm{YMLT}$ group predominantly appeared in 


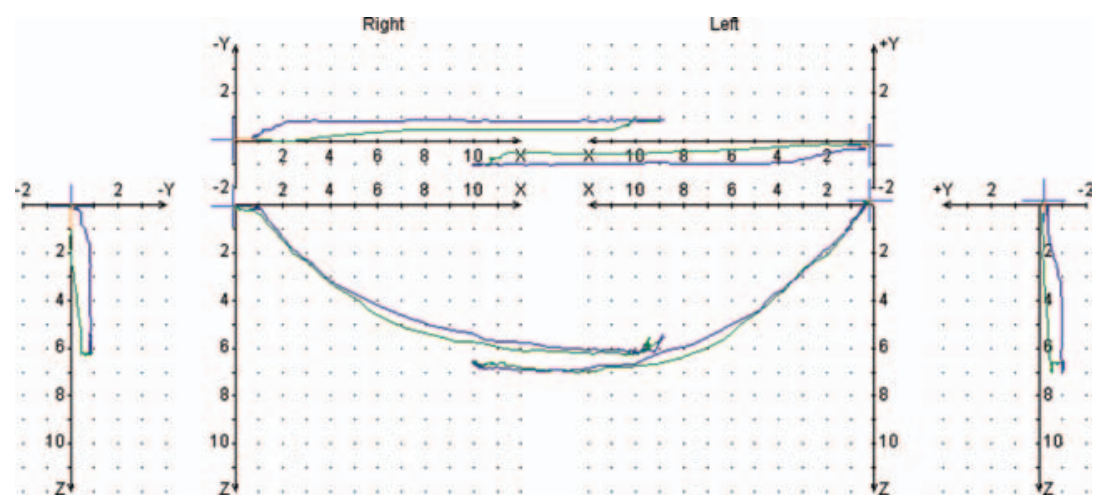

Fig. 3: Condylographic tracing; opening and closing movements represented $\triangle Y M L T$ to the left side. In closing movement, closing $\triangle Y M L T$ occurred at an approximately $2 \mathrm{~mm}$ anteriorly to RP in the right joint and $3 \mathrm{~mm}$ anteriorly to RP in the left joint. The tracing suggested that the $\Delta \mathrm{YMLT}$ suggests the latero-medial therapeutic position of the mandible right before the closing $\triangle Y M L T$ point

Tab. 1: Classification of translational side shift subjects $(n=62)$

\begin{tabular}{lcc}
\hline & Subject number & (\%) \\
\hline$\Delta Y$ shift group & $43 / 62$ & 69.4 \\
\hline Closed lock group & $12 / 62$ & 19.4 \\
\hline Excess rotation group & $7 / 62$ & 11.2 \\
\hline
\end{tabular}

antero-medial displacement (34.9\%). Antero-lateral displacement also showed relatively high frequency at $25.6 \%$ (Tab. 4).

The condyle tends to go in the direction of the displaced articular disk to "recapture" the disk. For example, in the case of antero-medial displacement of the disk, the condyle moves in an inward direction which makes $\triangle$ YMLT an inside directed shift. In order to investigate the relationship between the direction of $\Delta \mathrm{Y}$ and the type of disk displacement, coincidence in the direction of $\Delta \mathrm{Y}$ and the type of disk displacement were examined. As shown in the Tab. 5, quite high coincidence (95.3\%) was observed.

The $\triangle$ YMLT occurred in different combinations of articular disk displacement. Figures 4 and 5 shows combination of direction of $\Delta \mathrm{Y}$ and the type of disk displacement in subjects with reducible articular disk. High prevalence of $\triangle$ YMLT (32.6\%) was observed in case of combination of one side antero-medial (including medial sideway) and other side antero-lateral (including lateral sideway). The frequency of bilateral antero-medial and bilateral antero-lateral was $23.3 \%$ and $20.9 \%$, respectively.

\section{Three-dimensional mandibular movement and $\triangle Y M L T$}

There were two types of $\triangle \mathrm{YMLT}$ when the direction of $\triangle \mathrm{YMLT}$ and incisor shift (I shift) during opening movement was observed (Tab. 6). The first type showed coincidence in the directions of $\triangle$ YMLT and I shift. The second type did not coincide with these movements. Twenty-one of 40 joints (52.5\%) showed a coinciding pattern, while 15 of 40 joints (37.5\%) did not coincide.

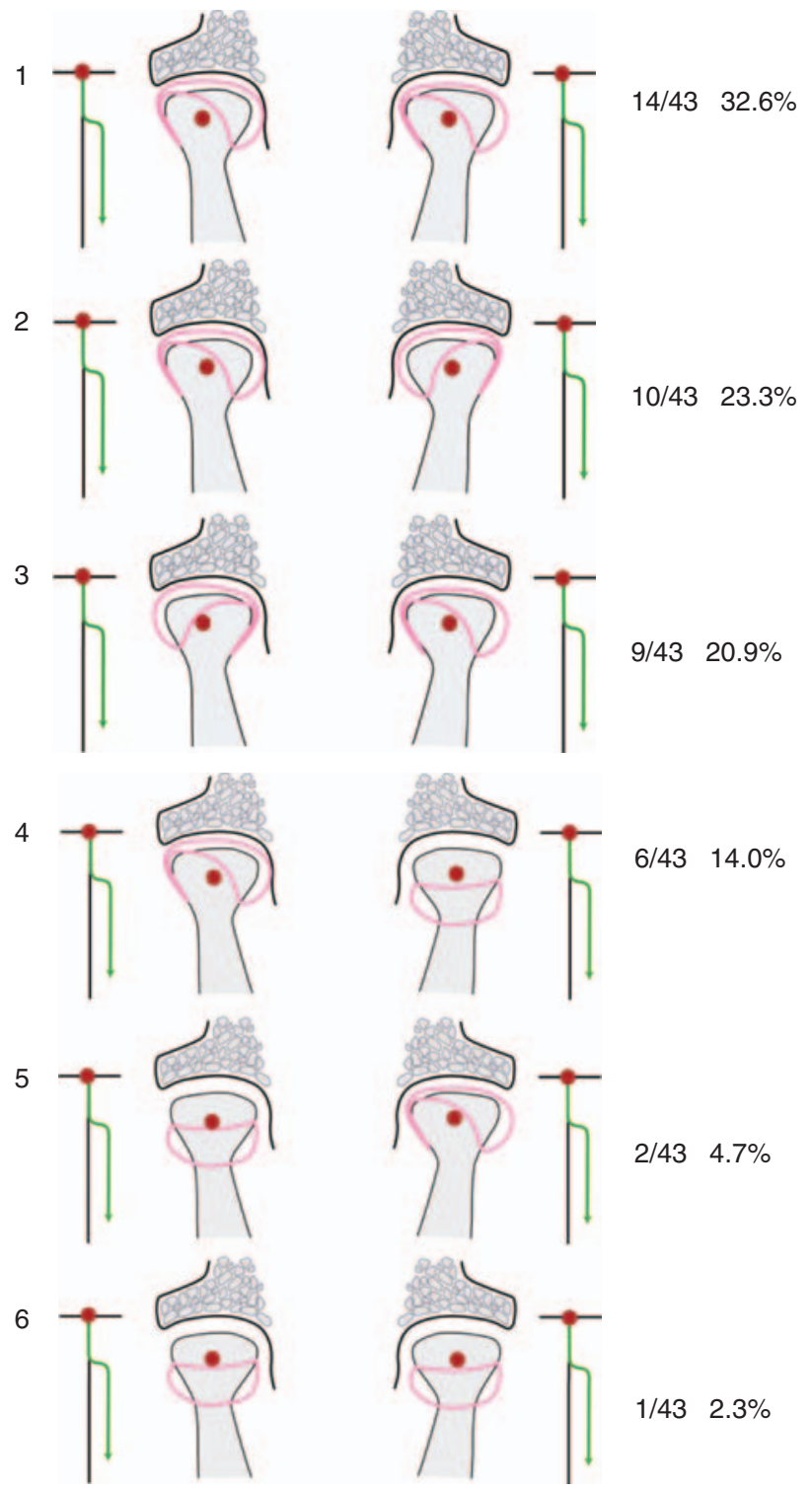

Fig. 4: Combination of disk displacement and direction of $\triangle$ YMLT. There were a variety of combinations of disk displacement in right and left joints. Approximately $77 \%$ of the cases showed the combination of lateral-medial displacement of articular disk 


\begin{tabular}{|c|c|c|c|c|}
\hline $\begin{array}{l}\text { Disk } \\
\text { displacement }\end{array}$ & $\begin{array}{l}\text { Number } \\
\text { of } \\
\text { subjects }\end{array}$ & $(\%)$ & $\begin{array}{l}\text { Number } \\
\text { of } \\
\text { joints }\end{array}$ & (\%) \\
\hline Unilateral reducible* & 16 & 26.2 & 16 & 13.1 \\
\hline Bilateral reducible & 33 & 54.1 & 66 & 54.1 \\
\hline Unilateral non-reducible* & 16 & 26.2 & 16 & 13.1 \\
\hline Bilateral non-reducible & 12 & 19.7 & 24 & 19.7 \\
\hline Total & 61 & & $122^{* *}$ & \\
\hline
\end{tabular}

Tab. 3: Reducibility of displaced articular disk in delta $Y$ subjects ( $n=43,86$ joints)

\begin{tabular}{lll} 
& Joint number & (\%) \\
\hline Reducible & $70 / 86$ & 81.4 \\
\hline Non-reducible & $16 / 86$ & 18.6 \\
\hline
\end{tabular}

Tab. 4: Distribution of articular disk displacement in delta $Y$ joint ( $n=43,86$ joints)

Number

of joints

\begin{tabular}{lcc}
\hline Complete anterior & 10 & 11.6 \\
\hline Antero-lateral & 22 & 25.6 \\
\hline Antero-medial & 30 & 34.9 \\
\hline Lateral sideway & 10 & 11.6 \\
\hline Medial sideway & 13 & 15.1 \\
\hline No displacement & 1 & 1.2 \\
\hline
\end{tabular}

Tab. 5: Coincidence of delta $Y$ direction and displacement of articular disk in delta $Y$ joint $(n=43)$

\begin{tabular}{lcr} 
& $\begin{array}{l}\text { Subject } \\
\text { number }\end{array}$ & (\%) \\
\hline Coincide & $41 / 43$ & 95.3 \\
\hline Not coincide & $2 / 43$ & 4.7 \\
\hline
\end{tabular}

Measurements of sagittal condylar inclination (SCI) at 5-mm translation $(\mathrm{S}=5 \mathrm{~mm})$ showed that SCI tended to be larger on the $\triangle$ YMLT side than that of the non- $\triangle$ YMLT side. This tendency was more obvious in the non-coinciding group than in the coinciding group (Tab. 7).
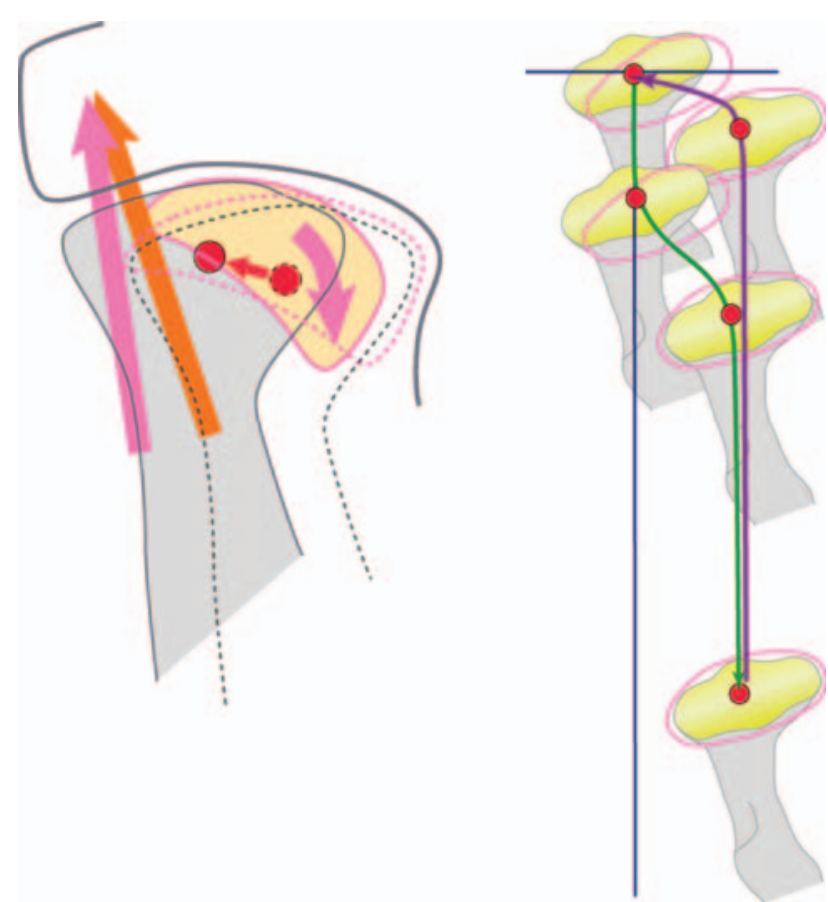

Fig. 5: Possible mechanism for the occurrence of $\triangle$ YMLT. Condylar displacement causes disk displacement and changes inter-relation of lateral pole of the condyle and lateral ligaments. These changes result in a secondary partially locked condyle between the displaced disk and lateral ligaments. The condylar lateral shift during symmetric movement ( $\triangle \mathrm{YMLT}$ ) offers escape from the partially locked situation

\section{Discussion}

In healthy TMJ, a posterior band of the disk covers the superior surface of the condyle, including the medial and lateral poles, and supports the condylar position vertically and medially when the mouth is closed. Therefore, disk displacement is considered to denote a lack of condylar support by the posterior band of the disk which indicates displacement of the condyle in the articular cavity when posterior occlusal support is decreased or lost. Condylar movement shows lateral deviation in order to obtain the normal disk-condylar relation when the mandible starts movement from a displaced position. The MLT in symmetric movements is one of the symptoms of intracapsular derangement of TMJ.

Results presented here showed that there were different types of MLT: $\triangle \mathrm{YMLT}$, closed lock MLT, and excessive rotation MLT. The $\triangle \mathrm{YMLT}$ was closely related with disunion of the disk-condyle assembly and reducibility of the displaced disk and condyle. Although approximately $20 \%$ of MLT subjects exhibited bilateral non-reducible disk displacement, predominant MLT appeared in the case which had bilateral or unilateral reducible disk displacement. The $\triangle \mathrm{YMLT}$, therefore, is suggested to "recapture" the displaced disk by the condyle in lower joint translation; this means condylar lateral movement towards the displaced disk. Evaluation of the coincidence between the directions of $\triangle \mathrm{YMLT}$ and disk displacement indicated $95.3 \%$ coincidence, which also confirmed the recapturing of the displaced disk by the condyle in lower joint translation.

There were two types of opening movements in $\triangle \mathrm{YMLT}$ subjects: mandibular bodily lateral translation which demon- 


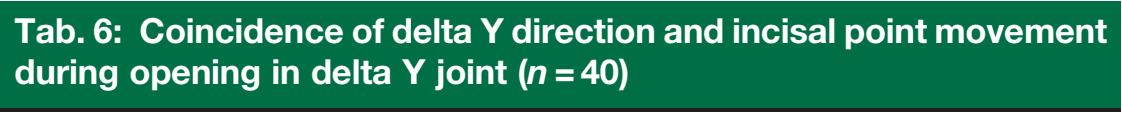

\begin{tabular}{lcc} 
& Subject number & (\%) \\
\hline Coincide & $21 / 40$ & 52.5 \\
\hline Not coincide & $15 / 40$ & 37.5 \\
\hline No-deviation & $4 / 40$ & 10.0 \\
\hline
\end{tabular}

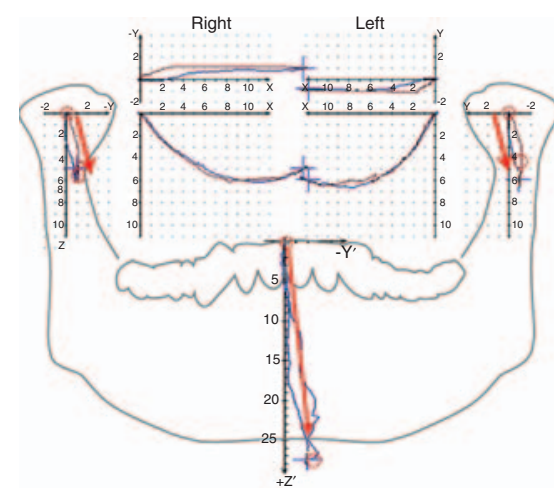

Tab. 7: Comparison of SCI between $\Delta Y$ and I shift coincide group and $\Delta Y$ and I shift non-coincide group $(n=40)$

\begin{tabular}{llll}
\hline & $\Delta \mathbf{Y}$ Side & $\begin{array}{l}\text { Non- } \Delta \mathbf{Y} \\
\text { side }\end{array}$ & Difference \\
\hline Total group $(n=40)$ & $54.1 \pm 11.7$ & $51.2 \pm 10.9$ & $2.9 \pm 8.6$ \\
\hline $\begin{array}{l}\Delta Y \text { and I shift coincide } \\
\text { group }(n=21)\end{array}$ & $55.3 \pm 12.2$ & $52.8 \pm 11.2$ & $2.5 \pm 8.2$ \\
\hline $\begin{array}{l}\Delta Y \text { and I shift } \\
\text { non-coincide } \\
\text { group }(n=15)\end{array}$ & $55.5 \pm 8.8$ & $48.9 \pm 11.8$ & $6.0 \pm 7.3$ \\
\hline
\end{tabular}

strated coincidence in the directions of $\triangle$ YMLT and I shift and mandibular rotational lateral translation which showed noncoincidence in the directions of $\triangle \mathrm{YMLT}$ and I shift. Both groups showed difference in SCI on the $\Delta \mathrm{Y}$ side and non- $\Delta$ Y side. The $\Delta \mathrm{Y}$ side of SCI had a larger value in both groups. Interestingly, the mandibular rotational lateral translation group ( $\Delta \mathrm{Y}$ and I shift non-coinciding group in Tab. 7 ) showed greater difference $(6.0 \pm 7.3)$ than that in the other.

Farrar and McCarty [5] stated that in patients with intracapsular derangement, the condyle tends to be located in the supero-posterior direction in the articular cavity when the teeth are in the intercuspal position. Serial sections of the MRI showed reduced space in the retro-articular region associated with anterior disk displacement and suggested that there was supero-posterior positioning of the condyle in a joint with internal derangement of TMJ [13]. In a patient who shows condylar deviation in its position within the articular cavity accompanied with anterior disk displacement, it is necessary to reposition the condyle anteriorly or inferiorly in order to obtain available space for the reduction of the disk. The new position of the condyle is referred to as the therapeutic position. It was reported that permanent maintenance of the

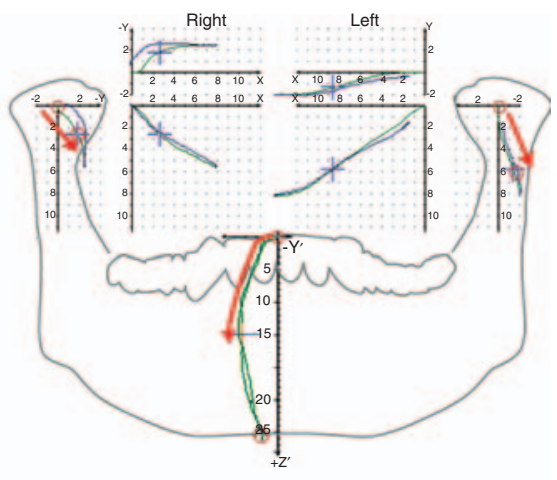

therapeutic position was important in order to achieve longterm success in patients with internal derangement treated using disk-repositioning appliances [11, 12].

Katzberg et al. [7] ${ }^{10)}$ and Liedberg et al. [10] had stated that the disk was not merely displaced anteriorly, but also rotational and sideways displacements of the TMJ disk were important aspects of intracapsular derangement. Therefore, it must be considered that condylar position is deviated three-dimensionally related to the displacement pattern of a disk. Although the therapeutic position of a condyle in the sagittal plane has been discussed, the considerations on the medio-lateral therapeutic position of a condyle have to be considered.

In this study, we classified disk displacement into five types: anterior, antero-medial, antero-lateral, medial sideway, and lateral sideway. The prevalence of antero-medial displacement was high (34.9\%), whereas those of antero-lateral and medial-sideway were $25.6 \%$ and $15.1 \%$, respectively. However, $\triangle$ YMLT seemed to be more related to the combination of disk displacement to the right and left sides. The combination of antero-medial and antero-lateral displacement showed higher prevalence. This result indicates that mandibular lateral displacement causes condylar displacement in the same direction, and disk displacement in the opposite direction, hence it creates $\triangle \mathrm{YMLT}$ which is oriented in the disk displacement direction. In cases of combined antero-lateral or anteromedial displacements, the direction of $\triangle \mathrm{YMLT}$ occurrence was probably dependent upon the progression of disunion and condition of the TMJ and associated to the structures such as muscles, ligaments, and SCI difference.

The significance of the assessment of the various patterns of disk displacement has been discussed by several investigators. On the basis of MRI findings, it was reported that $46 \%$ of joints with anterior disk displacement were accompanied by partial anterior disk displacement and MRI was more useful than arthrography in distinguishing between complete and partial displacement of the disk [8]. Katzberg et al. 
[7] investigated rotational and sideways disk displacement from MRI assessment and found that such disk displacements were present in $44 \%$ of cadaver joints and in $26 \%$ of joints of internal derangement patients. They also reported the applicability of MRI assessment on rotational and sideways disk displacement and stated that both sagittal and coronal images were necessary to diagnose such disk displacements.

The fact that the MLT occurs due to the reduction of the disk suggests that the condyle returned to the original and normal position medio-laterally in the mandibular fossa. In general, the displacement of the disk in a patient with disk displacement with reduction occurs at the end of closing and is associated with clicking. If so, the disk-condyle relationship is normal from the maximum opening to a position right before the closing click, and a straight closing path will be observed. On the basis of these findings, the therapeutic position in initial repositioning approach can be estimated as the condylar position immediately before the closing $\triangle$ YMLT.

Functions of the right and left TMJs are always interrelated. Dysfunction problem on one side can potentially affect the other side or a single injury of the jaw can affect both TMJs simultaneously. It has been reported that in patients with unilateral TMD, 95\% of joints in both symptomatic and asymptomatic sides were diagnosed with internal derangement based on arthrographic findings [9]. Therefore, it should be noted that bilateral joint problems are highly prevalent in TMJ patients, and even if a patient shows a unilateral joint symptom on clinical examination, close attention should always be paid to the opposite joint. From all these findings, it is recommended to make a correct diagnosis in such a complex system by incorporating a joint movement analyzing system including MLT for the early detection of intracapsular derangement.

\section{Take-home message}

The observations of MRI findings of $\triangle \mathrm{YMLT}$ show a close relationship between $\triangle$ YMLT and disk displacement. Therefore, it is recommended to make a correct diagnosis in dysfunction of the stomatognathic system by incorporating kinesiologic information including $\triangle$ YMLT for the early detection of intracapsular derangement.

\section{Acknowledgements}

This work was performed at the Research Institute of Occlusion Medicine and Research Center of Brain and Oral Science,
Kanagawa Dental College, and supported by Grants-in-Aid for Open Research from the Japanese Ministry of Education, Culture, Sports, Science and Technology.

\section{References}

[1] Clayton JA, et al. Graphic recordings of mandibular movements research criteria. J Prosthet Dent 1971;25:287-98.

[2] Clayton JA, et al. Pantographic tracings of mandibular movements and occlusion. J Prosthet Dent 1971;25:389-96.

[3] Dawson P. Centric relation: Chapter 4. In evaluation, diagnosis, and treatment of occlusal problems. St Louis, Mosby, 1989.

[4] Farrar WB. Characteristics of the condylar path in internal derangements of the TMJ. J Prosthet Dent 1978;39:319-23.

[5] Farrar WB, McCarty WL Jr. Inferior joint space arthrography and characteristics of condylar paths in internal derangements of the TMJ. J Prosthet Dent 1979;41:548-55.

[6] Fushima K, Sato S, Suzuki Y, et al. Horizontal condylar path in patients with disk displacement with reduction. J Craniomandib Pract 1994;12:78-86.

[7] Katzberg RW, Westesson PL, Tallents RH, et al. Temporomandibular joint: MR assessment of rotational and sideways disk displacements. Radiology 1988;169:741-8.

[8] Kerstens HCJ, Golding RP, Valk J, et al. Magnetic resonance imaging of partial temporomandibular joint disk displacement. J Oral Maxillofac Surg 1989;47:25-9.

[9] Kozeniauskas JJ, Ralph WJ. Bilateral arthrographic evaluation of unilateral temporomandibular joint pain and dysfunction. J Prosthet Dent 1988;60:98-105.

[10] Liedberg J, Westesson PL, Kurita K, et al. Sideways and rotational displacement of the temporomandibular joint disk: Diagnosis by arthrography and correlation to cryosectional morphology. Oral Surg Oral Med Oral Pathol1990;69:757-63.

[11] Lundh H, Westesson PL, Rune B, et al. Changes in the mandibular position during treatment with disk-repositioning onlays: a roentgen stereophotogrammetric study. Oral Surg Oral Med Oral Pathol 1988;65:657-62.

[12] Lundh H, Westesson PL, Jisander S, et al. Disk-repositioning onlays in the treatment of temporomandibular joint disk displacement: comparison with a flat occlusal splint and with no treatment. Oral Surg Oral Med Oral Pathol 1988;66:155-62.

[13] Marguelles-Bonnet R, Yung J-P, Carpentier P, et al. Temporomandibular joint serial sections made with mandible in intercuspal position, J Craniomandib Pract 1986;7:97-106.

[14] Mito T, Ishizaki K, Suzuki K, et al. Mandibular lateral translation during symmetrical mandibular movement. Int J Stomat Occl Med 2009;1:1-8.

[15] Paesani D, Westesson PL, Hatala M, et al. Prevalence of temporomandibular joint internal derangement in patients with craniomandibular disorders. Am J Orthod Dentofac Orthop1992;101:41-7.

[16] Piehslinger E, Celar AG, Celar RM, et al. Computerized axiography: Principles and methods. J Craniomandib Pract 1991;9:344-55.

[17] Sanchez-Woodworth RE, Tallents RH, Katzberg RW, et al. Bilateral internal derangements of temporomandibular joint: evaluation by magnetic resonance imaging. Oral Surg Oral Med Oral Pathol 1988;65:281-5.

[18] Slavicek R. Clinical and instrumental functional analysis for diagnosis and treatment planning. Part 5. Axiography. J Clin Orthod 1988;22:656-67.

[19] Slavicek R. Clinical and instrumental functional analysis for diagnosis and treatment planning. Part 7. Computer-aided axiography. J Clin Orthod 1988;22:776-87. 